\title{
Placental Growth Factor Promotes Cardiac Muscle Repair via Enhanced Neovascularization
}

\author{
Jianfeng Zhang ${ }^{\mathrm{a}, \mathrm{b}} \quad$ Anqing Chen ${ }^{\mathrm{a}}$ Yicheng Wu ${ }^{\mathrm{b}}$ Qiang Zhao \\ aDepartment of Cardiac Surgery, Ruijin Hospital, School of Medicine, Shanghai Jiao Tong University, \\ Shanghai, ' ${ }^{b}$ Department of Cardiovascular Surgery, Shanghai Chest Hospital, Shanghai Jiao Tong \\ University, Shanghai, China
}

\section{Key Words}

Mesenchymal stem cells (MSCs) - Cardiac muscle repair - Placental growth factor (PLGF) • Macrophage polarization

\begin{abstract}
Background/Aims: Transplantation of mesenchymal stem cells (MSCs) improves post-injury cardiac muscle repair using ill-defined mechanisms. Recently, we have shown that production and secretion of placental growth factor (PLGF) by MSCs play a critical role in the MSCsmediated post-injury cardiac muscle repair. In this study, we addressed the underlying molecular mechanisms, focusing specifically on the interactions between MSCs, macrophages and endothelial cells. Methods: We isolated macrophages (BM-MФ) from mouse bonemarrow derived cells based on F4/80 expression by flow cytometry. BM-M $\Phi$ were treated with different doses of PLGF. Cell number was analyzed by a MTT assay. Macrophage polarization was examined based on CD206 expression by flow cytometry. PLGF levels in macrophage subpopulations were analyzed by RT-qPCR and ELISA. Effects of macrophages on vascularization were evaluated by a collagen gel assay using Human umbilical vein endothelial cells (HUVECs) co-cultured with PLGF-treated macrophages. Results: PLGF did not increase macrophage number, but dose-dependently polarized macrophages into a M2 subpopulation. M2 macrophages expressed high levels of PLGF. PLGF-polarized M2 macrophages significantly increased tubular structures in the collagen gel assay. Conclusion: Our data suggest that MSCs-derived PLGF may induce macrophage polarization into a M2 subpopulation, which in turn releases more PLGF to promote local neovascularization for augmenting post-injury cardiac muscle repair. This study thus sheds novel light on the role of PLGF in cardiac muscle regeneration.
\end{abstract}

Qiang Zhao

or Anqing Chen

\section{KARGER 125}

Department of Cardiac Surgery, Ruijin Hospital, School of Medicine, Shanghai Jiao Tong University, 197 Ruijin Er Road, Shanghai 200025 (China)

E-Mail qiang_zhao14@163.com or zq11607@rjh.com.cn or E-Mail caq11608@rijh.com.cn 


\begin{tabular}{|c|c|c|}
\hline Cellular Physiology & Cell Physiol Biochem 2015;36:947-955 & \\
\hline and Biochemistry & $\begin{array}{l}\text { DOI: 10.1159/000430269 } \\
\text { Published online: June 12, } 2015\end{array}$ & $\begin{array}{l}\text { O) } 2015 \text { S. Karger AG, Basel } \\
\text { www.karger.com/cpb }\end{array}$ \\
\hline
\end{tabular}

\section{Introduction}

Mesenchymal stem cells (MSCs) have been shown to play potential roles in tissue regeneration [1-5]. Specifically, MSCs are known to promote neovascularization in ischemic myocardium to improve heart function [1-5]. However, the mechanisms underlying these processes remain unclear. Hence, further studies on the beneficial effects of MSCs on postinjury cardiac muscle repair are quite important.

The most common definition of macrophage activation has applied terms of M1 and M2 macrophages, primarily based on the differential effects of interleukin-4 (IL-4) and interferon gamma (IFN- $\gamma$ ) on the macrophage phenotype [6-10]. While M1 macrophages respond to T helper 1 lymphocytes and generate reactive oxygen species (ROS) and nitric oxide (NO) to destroy and eliminate pathogens and damaged cells, M2 macrophages respond to $\mathrm{T}$ helper 2 lymphocytes to mediate humoral immunity and tissue repair [6-12]. The processes that involve responses of macrophages to differential activation to adapt their phenotypes are called polarization [11, 12]. In vitro, bone-marrow-derived macrophages could be polarized to M1 or M2 macrophages using either IFN- $\gamma$, or IL-4, respectively [1113]. However, macrophages may adapt phenotypes in response to different stimulants, whereas the underlying mechanisms are not completely understood.

Recent studies have indicated an intimate relationship between MSCs and macrophages [14-18], and a role of placental growth factor (PLGF) on macrophage polarization [19-22]. These studies encouraged us to study the relationship between MSCs-derived PLGF and macrophage polarization in our recent published model, in which production and secretion of PLGF by MSCs have been found to be essential for the MSCs-mediated post-injury cardiac muscle repair [23]. Here, we further studied the underlying molecular mechanisms, paying special attention to the interactions among MSCs, macrophages and endothelial cells.

We isolated macrophages from mouse bone-marrow derived cells $(B M-M \Phi)$ based on F4/80 expression by flow cytometry. BM-M $\Phi$ were treated with different doses of PLGF. Cell number was quantified in an MTT assay. Macrophage polarization was examined based on CD206 expression on macrophages by flow cytometry. PLGF levels in macrophage subpopulations were analyzed by RT-qPCR and ELISA. The role of macrophages on vascularization was evaluated in a collagen gel assay using Human umbilical vein endothelial cells (HUVECs) co-cultured with polarized macrophages. We found that PLGF did not alter macrophage number, but dose-dependently polarized macrophages into a M2 subpopulation. M2 macrophages expressed high levels of PLGF. PLGF-induced M2 macrophages significantly increased tubular structures in the collagen gel assay. Thus, our data suggest that MSCsderived PLGF may induce macrophage polarization into a M2 subpopulation, which in turn releases more PLGF to promote local neovascularization to enhance post-injury cardiac muscle repair.

\section{Materials and Methods}

Mouse handling

All mouse experiments were approved by the Institutional Animal Care and Use Committee at Ruijin Hospital, Shanghai Jiaotong University School of Medicine (Animal Welfare Assurance). Male C57BL/6 mice (12-week-old) were used for isolation of bone-marrow cells in the current study.

Isolation and culture of bone-marrow derived mouse macrophages (BM-MФ) Isolation of bone-marrow derived mouse macrophages (BM-MФ) has been described before [24]. Mouse marrow was flushed out with vehicle solution (PBS containing 20mmol/l Tris and 100mmol/l NaCl, $\mathrm{pH}$ 7.5) through a 23G needle. Cells were pre-treated with FITC-conjugated F4/80 antibody (Becton-Dickinson Biosciences, San Jose, CA, USA) and then sorted for positive cells by flow cytometry. Purified F4/80-positive macrophages were cultured in Dulbecco's Modified Eagle Medium/F12 (DMEM/F12; Invitrogen, St. Louis, MO, USA) supplemented with $10 \mathrm{mmol} / \mathrm{l} \mathrm{L-glutamine,} 100 \mathrm{U} / \mathrm{ml}$ penicillin, $100 \mu \mathrm{g} / \mathrm{ml}$ streptomycin and $100 \mathrm{U} / \mathrm{ml}$ recombinant M-CSF (mouse M-CSF, R\&D Systems, Los Angeles, CA, USA). 


\section{Cellular Physiology Cell Physiol Biochem 2015;36:947-955 \begin{tabular}{l|l|l}
\hline DOI: 10.1159/000430269 & C 2015 S. Karger AG, Basel
\end{tabular} \begin{tabular}{l|l} 
and Biochemistry Published online: June 12, 2015 & www.karger.com/cpb
\end{tabular} \\ Zhang et al.: PLGF Promotes Cardiac Neovascularization}

\section{Fluorescence-activated cell sorting (FACS) for macrophages}

Cultured cells were detached with $0.25 \%$ Trypsine solution (Invitrogen), washed three times with PBS, re-suspended, labeled with FITC-conjugated F4/80 antibody and/or PEcy7-conjugated CD206 antibody (Becton-Dickinson Biosciences), for sorting for macrophages, or M1 and M2 subtypes. Flow cytometry was performed in a FACSAria flow cytometer (Becton-Dickinson Biosciences). Data were analyzed and quantified using Flowjo software (Flowjo LLC, Ashland, OR, USA).

MTT assay

For assay of cell growth, cells were seeded into 24 well-plate at $10^{4}$ cells per well in the conditioned media and subjected to an MTT Kit (Roche, Indianapolis, IN, USA), according to the instruction of the manufacturer. The MTT assay is a colorimetric assay for assessing viable cell number, since NAD(P) $\mathrm{H}$-dependent cellular oxidoreductase enzymes in viable cells are capable of reducing the tetrazolium dye MTT 3-(4,5-dimethylthiazol-2-yl)-2,5-diphenyltetrazolium bromide to its insoluble formazan, which is in purple color and could be quantified by analyzing the absorbance value (OD) of each well at $540 \mathrm{~nm}$. Experiments were performed three times.

\section{Quantitative real-time PCR (RT-qPCR)}

RNA was extracted from cultured cells with RNeasy (Qiagen, Hilden, Germany) for cDNA synthesis. Complementary DNA (cDNA) synthesis was performed by reserve transcription. Quantitative PCR (RTqPCR) was performed in duplicates with QuantiTect SYBR Green PCR Kit (Qiagen). All primers were purchased from Qiagen. Quantification used ddCT method. Values of genes were first normalized against $\beta$-actin, and then compared to controls.

\section{ELISA}

The secreted PLGF was determined by a PLGF ELISA Kit (R\&D System, Los Angeles, CA, USA). ELISA was performed according to the instructions of the manufacturer. Briefly, the collected condition media were added to a well coated with PLGF polyclonal antibody, and then immunosorbented by biotinylated monoclonal anti-human PLGF antibody at room temperature for 2 hours. The color development catalyzed by horseradish peroxidase was terminated with $2.5 \mathrm{~mol} / \mathrm{l}$ sulfuric acid and the absorption was measured at $450 \mathrm{~nm}$. The protein concentration was determined by comparing the relative absorbance of the samples with the standards.

\section{Collagen gel assay and quantification of vessel formation}

HUVECs were obtained from American Type Culture Collection (ATCC, Rockville, MD, USA), and cultured in Dulbecco's Modified Eagle Medium/F12 (DMEM/F12; Invitrogen) supplemented with 10mmol/l L-glutamine, $100 \mathrm{U} / \mathrm{ml}$ penicillin, $100 \mu \mathrm{g} / \mathrm{ml}$ streptomycin, $20 \%$ fetal bovine serum (FBS, Invitrogen) and endothelial cell growth supplement (ECGS, Becton-Dickinson Biosciences) at $37^{\circ} \mathrm{C}$ and $5 \% \mathrm{CO}_{2}$. $\mathrm{HUVEC}$ between P3 and P4 were used for all experiments. The collagen gel assay was done based on established protocol. Briefly, wells of a six-well tissue culture plate were coated with $1.5 \mathrm{mg} / \mathrm{ml}$ of rat tail collagen type 1 and $3 \mu \mathrm{g} / \mathrm{ml}$ of fibronectin (Becton-Dickinson Biosciences). HUVECs (5 X 10 $10^{5}$ ) were seeded in each collagen-coated well and allowed to attach for $1 \mathrm{~h}$. A second collagen layer was added to a well and allowed to polymerize. HUVECs were then co-cultured with PLGF-treated macrophages in a transwell. Five random images were taken from each experimental conditions, and analyzed by NIH ImageJ. Number of sprouts per bead was determined, where a sprout is defined as a vessel of length equal to the diameter of a bead. Sprout length was also measured in arbitrary units. For statistical analysis, 25 beads were assessed for each condition.

\section{Statistics}

GraphPad Prism software (GraphPad Software, Inc. La Jolla, CA, USA) was used for statistical analyses. Unpaired two-tailed Student $t$ test was applied for comparison between two groups, and one-way ANOVA with the Tukey posttest was applied for comparison between three or more groups. Data were represented as mean $\pm \mathrm{SD}$ and were considered significant if $\mathrm{p}<0.05$. 


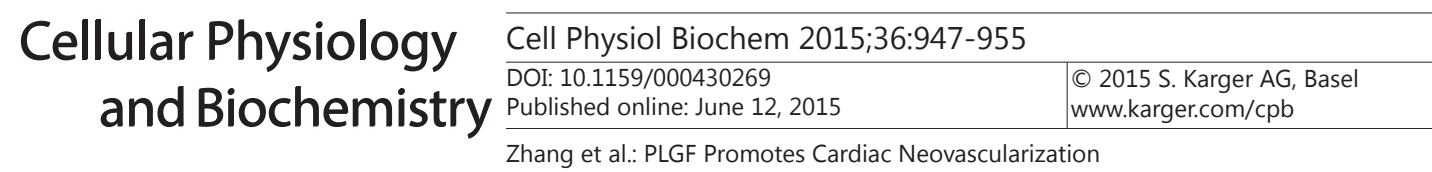

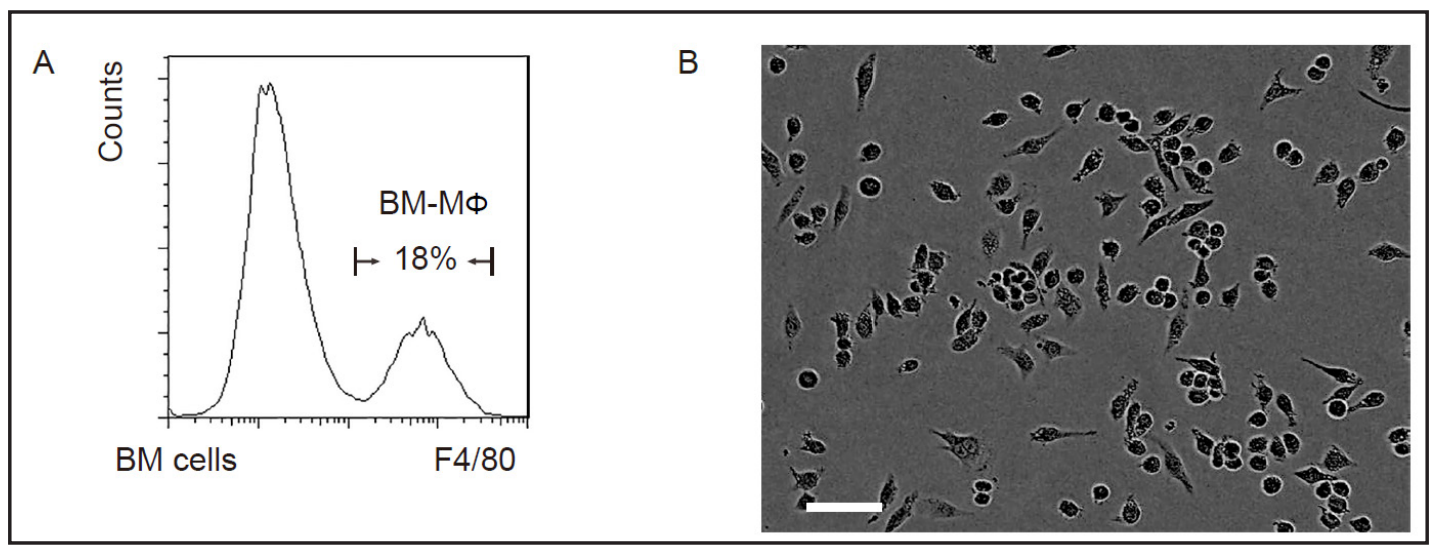

Fig. 1. Isolation and differentiation of bone-marrow-derived macrophages. (A) We isolated bone marrow cells from mice and then purified macrophages from bone-marrow derived cells (BM-MФ) based on F4/80 expression by flow cytometry, shown by a presentative flow chart. (B) Purified BM-M $\Phi$ in culture. Scale bar is $20 \mu \mathrm{m}$.

\section{Results}

Isolation and differentiation of mouse bone-marrow-derived macrophages

First, we isolated mouse bone-marrow cells and then purified macrophages (BM-M $\Phi)$ based on F4/80 expression by flow cytometry (Fig. 1A). The purified BM-M $\Phi$ were then kept in culture (Fig. 1B).

PLGF dose-dependently polarizes BM-MФ into a M2 subtype

Then we treated cultured BM-M $\Phi$ with different doses (low dose: $1 \mathrm{nmol} / \mathrm{l}$; medium dose: $5 \mathrm{nmol} / \mathrm{l}$; high dose: $25 \mathrm{nmol} / \mathrm{l}$ ) of PLGF (Fig. 2A). We examined whether PLGF may alter the viability of BM-MФ in an MTT assay. We found that PLGF did not change the cell number of cultured BM-MФ 4 days after PLGF treatment (Fig. 2B). Then, we examined whether PLGF may alter macrophage polarization by flow cytometry using a M2-macrophage-specific marker CD206. We found that PLGF dose-dependently polarized BM-MФ into a M2 subtype, by representative flow charts (Fig. 2C), and by quantification (Fig. 2D). These data suggest that PLGF dose-dependently polarizes BM-M $\Phi$ into a M2 subtype.

M2 macrophages produce high levels of PLGF

We has shown that PLGF promotes neovascularization in MSCs-mediated cardiac muscle repair [23]. Thus, we aimed to find out whether macrophage polarization may change its PLGF production. We purified M1 and M2 macrophage based on expression of CD206 by flow cytometry. Then we analyzed gene expression in M1 and M2 subpopulations, after confirmation of the purity of M1 and M2 macrophages based on the transcript level of a M1 marker iNOS and a M2 marker Arginase (Fig. 3A). While we did not find any changes in these genes in the M1 or M2 macrophages, we found that M2 macrophages expressed significantly higher PLGF transcripts (Fig. 3A) and secreted significantly more PLGF (Fig. 3B), compared to M1 macrophages. These data suggest that M2 macrophages produce high PLGF, and may contribute to the MSCs-mediated cardiac muscle repair.

PLGF-polarized M2 macrophages promote neovascularization in vitro

In order to confirm that PLGF-polarized M2 macrophages may promote neovascularization, we applied a collagen gel assay using HUVEC, in which HUVEC cells were cultured in a transwell with the identical number of macrophages polarized by different doses of PLGF (Fig. 4A). In this model, macrophages were first treated with different doses of PLGF to induce M2 polarization, and then the cultured media were removed to avoid the 
Cellular Physiology Cell Physiol Biochem 2015;36:947-955

and Biochemistry Pullished online: June 12, 2015

Zhang et al.: PLGF Promotes Cardiac Neovascularization

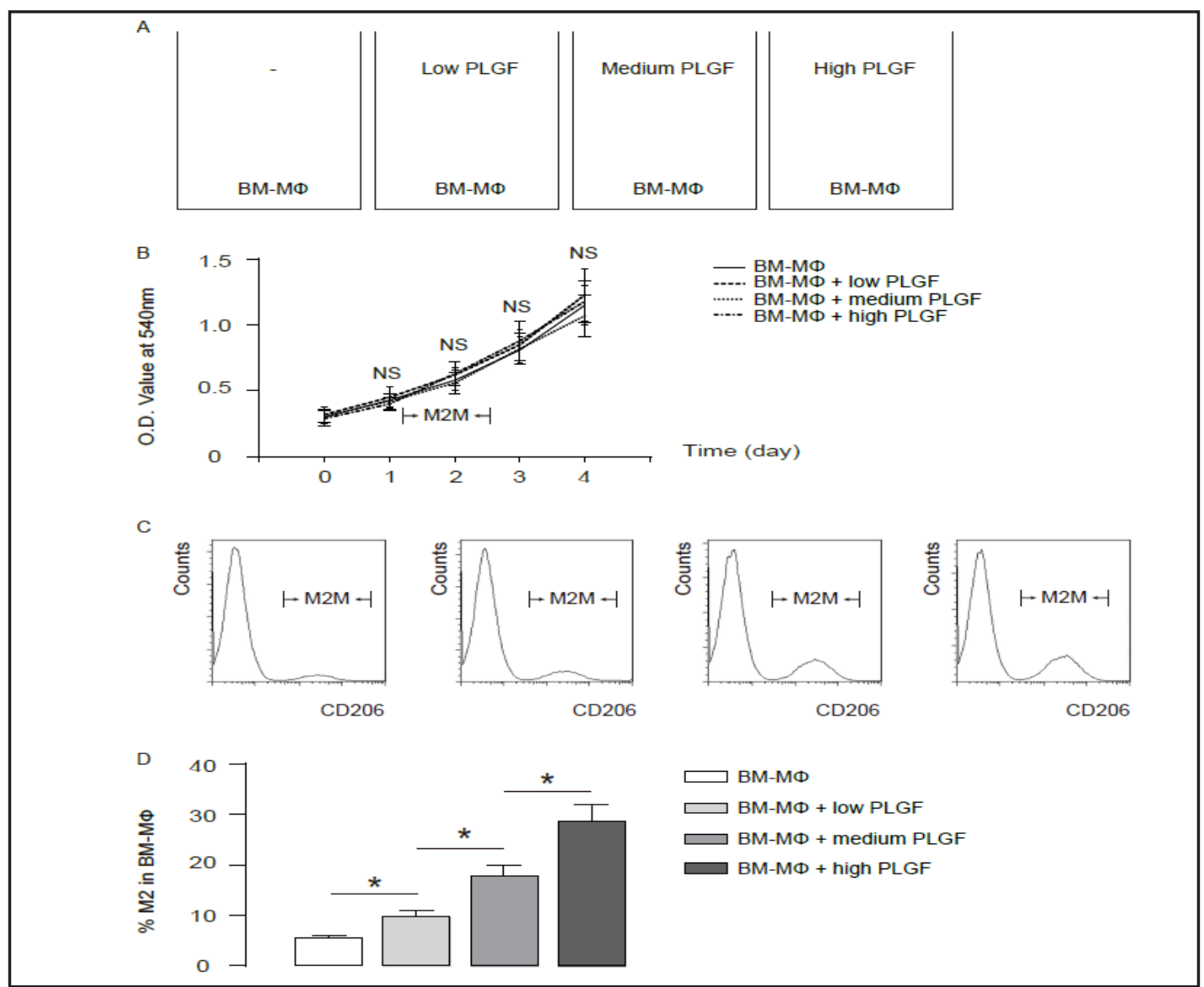

Fig. 2. PLGF dose-dependently polarizes BM-MФ into a M2 subtype. (A) We treated BM-M $\Phi$ with different doses (low dose: $1 \mathrm{nmol} / \mathrm{l}$; medium dose: $5 \mathrm{nmol} / \mathrm{l}$; high dose: $25 \mathrm{nmol} / \mathrm{l}$ ) of PLGF in culture. (B) The viability of BM-M $\Phi$ by PLGF was examined in an MTT assay. (C-D) Macrophage polarization was examined by flow cytometry using a M2-macrophage-specific marker CD206, shown by representative flow charts (C), and by quantification (D). ${ }^{*} p<0.05$. NS: non-significant. $N=5$.

Fig. 3. M2 macrophages produce high PLGF. (AB) $\mathrm{M} 1$ and $\mathrm{M} 2$ $\mathrm{m}$ acrophages were purified by flow cytometry, and used for gene expression analyses (A) and secreted PLGF analyses by ELISA (B). ${ }^{*} \mathrm{p}<0.05$. NS: non-significant. $\mathrm{N}=5$.

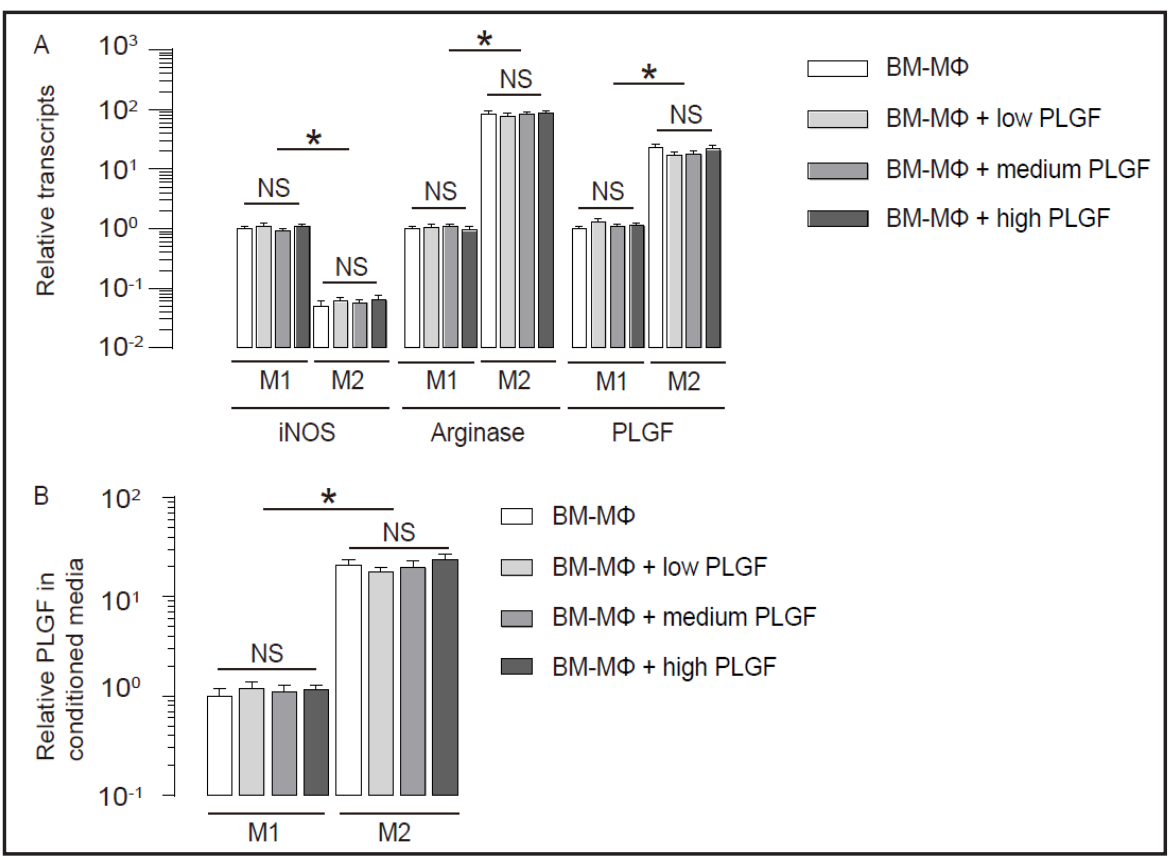


Fig. 4.

P L G F - p o larized M2 macrophages promote neovascu larization in vitro. (A) The effects of PLGF-polarized M2 macrophages on neovascularization were examined in a collagen gel assay using HUVEC. In this model, first, macrophages were treated with different doses of PLGF to induce M2 polarization, and then the cultured media were removed to avoid the effects of the exogenous PLGF on HUVECs. Afterwards, the polarized macrophages were co-cultured
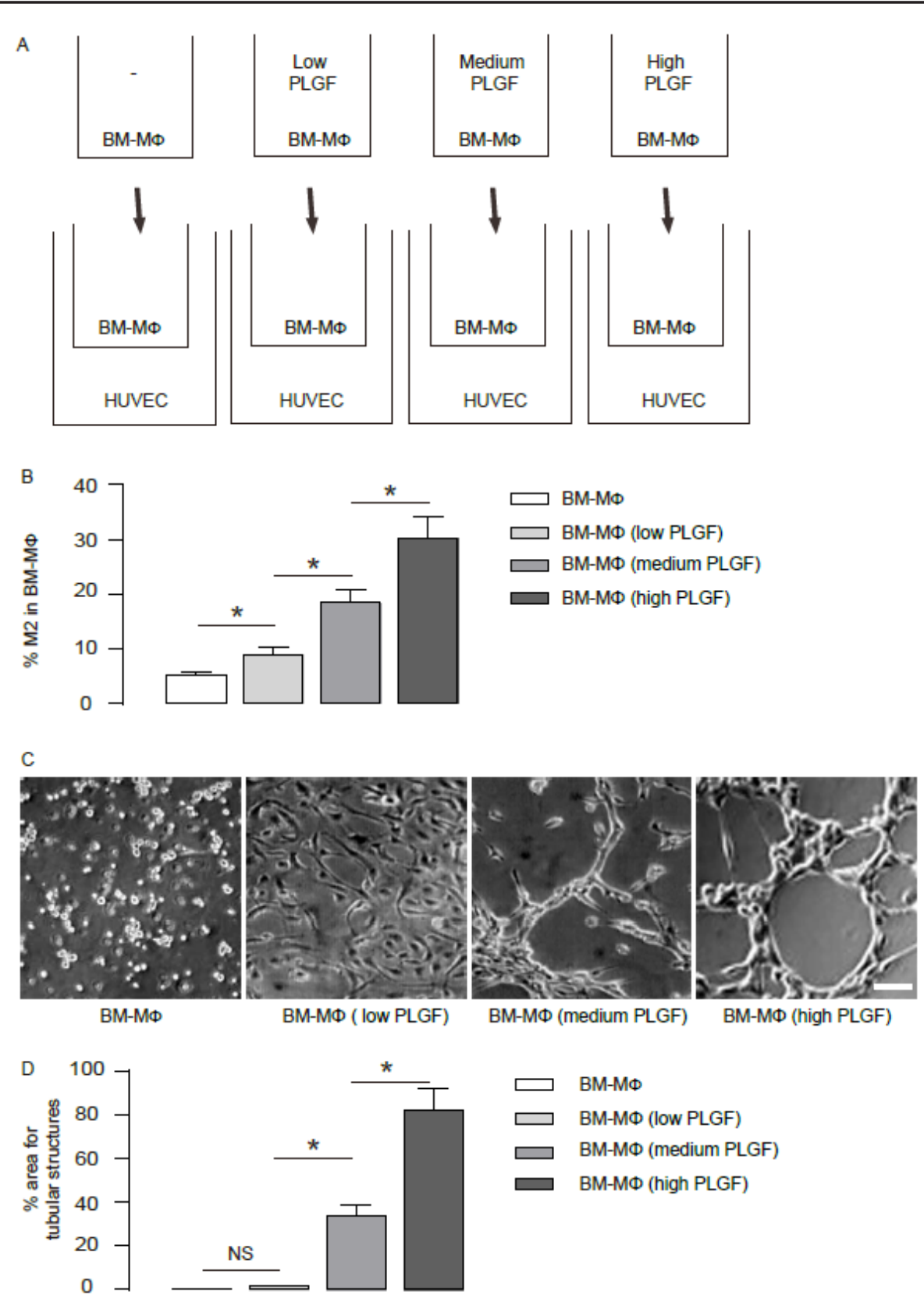

with HUVECs in a transwell and the tubular structure formation was examined after 7 days. (B) M2 macrophage polarization was confirmed by flow cytometry, shown by quantification. (C-D) HUVEC tubular structure formation by M2-polarized macrophages was evaluated and shown by representative images (C), and by quantification (D). * ${ }^{*}<0.05$. NS: non-significant. $\mathrm{N}=5$. Scale bar is $30 \mu \mathrm{m}$.

effects of the exogenous PLGF on HUVECs. Afterwards, the polarized macrophages were cocultured in a transwell with HUVECs at a ratio of 1:1. After 7 days, the tubular structures was quantified (Fig. 4A).

First, M2 macrophage polarization was examined by flow cytometry, showing that macrophages that were treated with higher doses of PLGF contained more M2 subtype (Fig. 4B). Then, we found that M2 macrophages significantly increased tubular structure formation in HUVEC cells in the collagen gel assay, by representative images (Fig. 4C), and by

\section{KARGER}


Fig. 5. Schematic of the model. MSCs-derived PLGF may induce macrophage polarization into a M2 subpopulation, which in turn releases more PLGF to promote local neovascularization to enhance post-injury cardiac muscle repair.

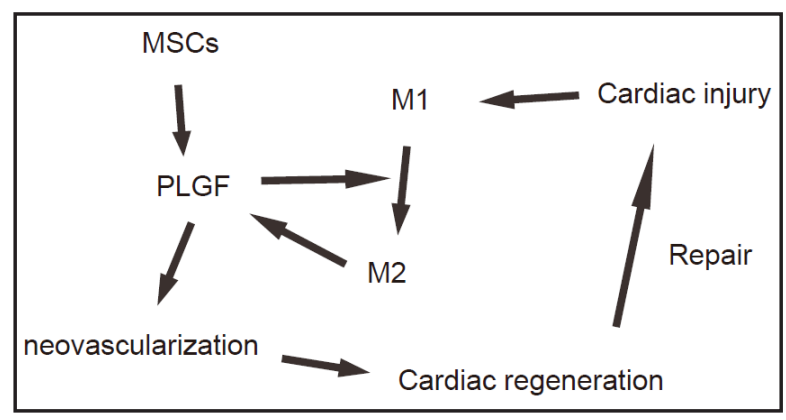

quantification (Fig. 4D). Together, these data suggest that PLGF-polarized M2 macrophages promote neovascularization in vitro.

Based on our findings in the current study and our previous work [23], we propose a model in which MSCs-derived PLGF may induce macrophage polarization into a M2 subpopulation, which in turn releases more PLGF to promote local neovascularization to enhance post-injury cardiac muscle repair (Fig. 5).

\section{Discussion}

Transplantation of MSCs has established therapeutic effects on cardiac muscle repair after injury. However, previous studies have shown some controversial results. Moreover, the studies on the underlying mechanisms are ill-defined [3-5, 25]. Hence, we have recently studied this question, using both in vivo and in vitro models.

In our previous work, we isolated and cultured primary mouse MSCs, and assured the phenotypes of MSCs using classical differentiation assays. In order to evaluate the effects of MSCs transplantation on the recovery and regeneration of the injured mouse heart, we transplanted MSCs immediately after induction of acute myocardial infarction, which significantly improved cardiac muscle repair and heart function. We further show that the direct contribution of MSCs to regenerated cardiac muscle cells is very limited, and MSCs appear to promote neovascularization through secreting PLGF, but not VEGF-A. These data are consistent with an essential role of PLGF in pathological vascularization [26], and highlights PLGF as a key factor during cardiac muscle repair. However, the number of MSCs detected in the injured mouse heart is very modest. Therefore, the PLGF from MSCs may not be the major source of PLGF in the injured heart, and may be just an initiator for PLGF production. Hence, we hypothesize that MSCs may interact with macrophages to mediate the effects on neovascularization.

In this study, we tried to prove this hypothesis. First, we isolated bone-marrow cells from mice and then purified macrophages (BM-MФ) from bone-marrow derived cells. We found that PLGF did not change the cell number of macrophages 4 days after PLGF treatment. These data suggest that PLGF may not alter macrophage apoptosis or proliferation. Hence, it is expected that PLGF may change the polarization of macrophages. Indeed, we found that PLGF dose-dependently polarized BM-M $\Phi$ into a M2 subtype, and most interestingly, M2 macrophages produced about 50 times more PLGF than M1 macrophages. These data suggest that transplanted MSCs may secrete PLGF to polarize macrophages, which subsequently secrete more PLGF.

To confirm a role of M2 macrophages in neovascularization, we used a collagen gel assay, in which HUVEC cells were cultured in a transwell with identical number of macrophages polarized by different doses of PLGF. We found that M2 macrophages significantly increased tubular structure formation in HUVEC cells. Together, these data suggest that PLGF-polarized M2 macrophages promote neovascularization in vitro.

Based on our findings in the current study and our previous work [23], we propose a model in which MSCs-derived PLGF may induce macrophage polarization into a M2 


\section{Cellular Physiology Cell Physiol Biochem 2015;36:947-955 \\ \begin{tabular}{c|c|}
\hline DOI: 10.1159/000430269 & O 2015 S. Karger AG, Basel \\
\hline
\end{tabular} \\ www.karger.com/cpb \\ Zhang et al.: PLGF Promotes Cardiac Neovascularization}

subpopulation, which in turn releases more PLGF to promote local neovascularization to enhance post-injury cardiac muscle repair. This study thus sheds novel light on the role of PLGF in cardiac muscle regeneration.

\section{Disclosure Statement}

None disclosed.

\section{Reference}

1 Losordo DW, Dimmeler S: Therapeutic angiogenesis and vasculogenesis for ischemic disease: Part ii: Cellbased therapies. Circulation 2004;109:2692-2697.

2 Losordo DW, Dimmeler S: Therapeutic angiogenesis and vasculogenesis for ischemic disease. Part i: Angiogenic cytokines. Circulation 2004;109:2487-2491.

3 Murry CE, Soonpaa MH, Reinecke H, Nakajima H, Nakajima HO, Rubart M, Pasumarthi KB, Virag JI, Bartelmez SH, Poppa V, Bradford G, Dowell JD, Williams DA, Field LJ: Haematopoietic stem cells do not transdifferentiate into cardiac myocytes in myocardial infarcts. Nature 2004;428:664-668.

4 Balsam LB, Wagers AJ, Christensen JL, Kofidis T, Weissman IL, Robbins RC: Haematopoietic stem cells adopt mature haematopoietic fates in ischaemic myocardium. Nature 2004;428:668-673.

5 Fukata M, Ishikawa F, Najima Y, Yamauchi T, Saito Y, Takenaka K, Miyawaki K, Shimazu H, Shimoda K, Kanemaru T, Nakamura K, Odashiro K, Nagafuji K, Harada M, Akashi K: Contribution of bone marrowderived hematopoietic stem/progenitor cells to the generation of donor-marker(+) cardiomyocytes in vivo. PLoS One 2013;8:e62506.

6 Gordon S: Alternative activation of macrophages. Nat Rev Immunol 2003;3:23-35.

7 Gordon S, Martinez FO: Alternative activation of macrophages: Mechanism and functions. Immunity 2010;32:593-604.

8 Geissmann F, Manz MG, Jung S, Sieweke MH, Merad M, Ley K: Development of monocytes, macrophages, and dendritic cells. Science 2010;327:656-661.

9 Ricardo SD, van Goor H, Eddy AA: Macrophage diversity in renal injury and repair. J Clin Invest 2008;118:3522-3530.

10 Xiao X, Gaffar I, Guo P, Wiersch J, Fischbach S, Peirish L, Song Z, El-Gohary Y, Prasadan K, Shiota C, Gittes GK: M2 macrophages promote beta-cell proliferation by up-regulation of smad7. Proc Natl Acad Sci U S A 2014;111:E1211-1220.

11 Mills CD: M1 and m2 macrophages: Oracles of health and disease. Crit Rev Immunol 2012;32:463-488.

12 Mills CD, Kincaid K, Alt JM, Heilman MJ, Hill AM: M-1/m-2 macrophages and the th1/th2 paradigm. J Immunol 2000;164:6166-6173.

13 Jia RZ, Ding GC, Gu CM, Huang T, Rui C, Wang YX, Lu Q: Cdx2 enhances htr-8/svneo trophoblast cell invasion by altering the expression of matrix metalloproteinases. Cell Physiol Biochem 2014;34:628-636.

14 Li B, Shao Q, Ji D, Li F, Chen G: Mesenchymal stem cells mitigate cirrhosis through bmp7. Cell Physiol Biochem 2015;35:433-440.

15 Liu W, Zhang S, Gu S, Sang L, Dai C: Mesenchymal stem cells recruit macrophages to alleviate experimental colitis through tgfbeta1. Cell Physiol Biochem 2015;35:858-865.

16 Pan B, Liu G, Jiang Z, Zheng D: Regulation of renal fibrosis by macrophage polarization. Cell Physiol Biochem 2015;35:1062-1069.

17 Song X, Xie S, Lu K, Wang C: Mesenchymal stem cells alleviate experimental asthma by inducing polarization of alveolar macrophages. Inflammation 2015;38:485-492.

18 Cao X, Han ZB, Zhao H, Liu Q: Transplantation of mesenchymal stem cells recruits trophic macrophages to induce pancreatic beta cell regeneration in diabetic mice. Int J Biochem Cell Biol 2014;53:372-379.

19 Rolny C, Mazzone M, Tugues S, Laoui D, Johansson I, Coulon C, Squadrito ML, Segura I, Li X, Knevels E, Costa S, Vinckier S, Dresselaer T, Akerud P, De Mol M, Salomaki H, Phillipson M, Wyns S, Larsson E, Buysschaert I, Botling J, Himmelreich U, Van Ginderachter JA, De Palma M, Dewerchin M, Claesson-Welsh L, Carmeliet P: Hrg inhibits tumor growth and metastasis by inducing macrophage polarization and vessel normalization through downregulation of plgf. Cancer Cell 2011;19:31-44. 


\section{Cellular Physiology Cell Physiol Biochem 2015;36:947-955 \begin{tabular}{l|l|l|}
\hline DOI: 10.1159/000430269 & (0) 2015 S. Karger AG, Basel
\end{tabular} \\ Zhang et al.: PLGF Promotes Cardiac Neovascularization}

20 Fischer C, Jonckx B, Mazzone M, Zacchigna S, Loges S, Pattarini L, Chorianopoulos E, Liesenborghs L, Koch M, De Mol M, Autiero M, Wyns S, Plaisance S, Moons L, van Rooijen N, Giacca M, Stassen JM, Dewerchin M, Collen D, Carmeliet P: Anti-plgf inhibits growth of vegf(r)-inhibitor-resistant tumors without affecting healthy vessels. Cell 2007;131:463-475.

21 Pavlov OV, Sheveleva TS, Selkov SA: In vitro expression of vascular endothelial growth factor and its receptors by placental macrophages. Bulletin of experimental biology and medicine 2012;153:222-225.

22 Adini A, Kornaga T, Firoozbakht F, Benjamin LE: Placental growth factor is a survival factor for tumor endothelial cells and macrophages. Cancer Res 2002;62:2749-2752.

23 Zhang J, Wu Y, Chen A, Zhao Q: Mesenchymal stem cells promote cardiac muscle repair via enhanced neovascularization. Cell Physiol Biochem 2015;35:1219-1229.

24 Weischenfeldt J, Porse B: Bone marrow-derived macrophages (bmm): Isolation and applications. CSH protocols 2008;2008:pdb prot5080.

$25 \mathrm{Wu}$ Y, Zhao RC, Tredget EE: Concise review: Bone marrow-derived stem/progenitor cells in cutaneous repair and regeneration. Stem Cells 2010;28:905-915.

26 Autiero M, Waltenberger J, Communi D, Kranz A, Moons L, Lambrechts D, Kroll J, Plaisance S, De Mol M, Bono F, Kliche S, Fellbrich G, Ballmer-Hofer K, Maglione D, Mayr-Beyrle U, Dewerchin M, Dombrowski S, Stanimirovic D, Van Hummelen P, Dehio C, Hicklin DJ, Persico G, Herbert JM, Shibuya M, Collen D, Conway EM, Carmeliet P: Role of plgf in the intra- and intermolecular cross talk between the vegf receptors flt 1 and flk1. Nat Med 2003;9:936-943. 